\title{
"Epidemiological survey of benign oral lesions in the Oral and Maxillofacial Surgery Area of the Piracicaba Dental School"
}

\section{Giulia Antonieto da Silva*, Márcio de Moraes}

\begin{abstract}
The aim of this epidemiological survey was to describe the distribution and understand the epidemiologic of the oral lesions treated in the Oral and Maxillofacial Surgery (OMFS) Area - School of Dentistry of Piracicaba - Unicamp (FOPUnicamp), in order to obtain information with sufficient scientific evidence to the epidemiological profile studied. The methodology used was the review of 786 clinical records, the data disclosed in the software developed and subsequent epidemiological study. As a result, most of the lesions were benign. The three most common oral lesions detected were Odontogenic Keratocyst, dentigerous cyst and traumatic bone cyst.
\end{abstract}

\section{Key words:}

Epidemiology, oral lesions, oral pathology

\section{Introduction}

The epidemiology investigation and the occurrence of oral diseases have become an important tool for understand the prevalence, extent and degree of oral diseases in the general population (Gheno JN et al,.2015).

Regarding, therefore, the importance of epidemiological studies, it is opportune to evaluate and review the diagnoses obtained in the OMFS Area - FOP / UNICAMP and obtain the most frequent epidemiological indexes and oral pathologies in our local area.

\section{Results and Discussion}

From the total of 786 clinical records, from June 1996 to June 2019, it was possible to trace an epidemiological profile in the considered period.

The cases affected all decades of life, with a mean age was 35.57 years.

Table 1. Gender of the patients with Oral Lesions ( $\mathrm{n}=$ 786)

\begin{tabular}{cll}
\hline Gender & Number & Percent \\
Female & 402 & 51.14 \\
Male & 384 & 48.59 \\
\hline
\end{tabular}

Table 2. Frequency of habits

Habits

\section{Number Percent}

$\begin{array}{lll}\text { Present } & 281 & 35.75 \\ \text { Absent } & 505 & 64.24\end{array}$

Table 3. Frequency of smoker, non-smoker and x-smokers

\begin{tabular}{cll}
\hline Smoking & Number & Percent \\
Non-Smoker & 617 & 78.49 \\
Smoker & 123 & 15.64 \\
X-smokers & 46 & 5.85 \\
\hline
\end{tabular}

Table 4. Frequency of alcohol

\begin{tabular}{cll}
\hline Alcohol & Number & Percent \\
No & 670 & \\
Yes & 100 & 85.24 \\
x-trader & 13 & 12.72 \\
\hline
\end{tabular}

Table 5. Most frequent oral lesions

\begin{tabular}{lll}
\hline Diagnostic & Number & Percent \\
Keratocyst & 70 & 8.90 \\
Dentigerous cyst & 39 & 4.96 \\
Traumatic bone cyst & 30 & 3.81 \\
\hline
\end{tabular}

\section{Conclusions}

The highest frequency of the Odontogenic Keratocyst is in accord previous study in our area, but many other lesions as has been shown in this study is in agreement with other studies in the literature (SiguaRodriguez EA et al,.2019).

\section{Acknowledgement}

\section{PIBIC}

Sigua-Rodriguez EA, Goulart DR, Sverzut A, Asprino L, de Moraes M. Is Surgical Treatment Based on a 1-Step or 2-Step Protocol Effective in Managing the Odontogenic Keratocyst? J Oral Maxillofac Surg. 2019 Jun;77(6):1210.e11210.e7. doi: 10.1016/j.joms.2019.02.040. Epub 2019 Mar 5.

Gheno JN, Martins MAT, Munerato MC, Hugo FN, Sant'Ana Filo M, Weissheimer $\mathrm{C}$, et al. Oral mucosal lesions and their association with sociodemographic, behavioral, and health status factors. Braz Oral Res. 2015;29:1-6.

Kansky, A., Didanovic, V., Dovsak, T., Brzak, B., Pelivan, I., \& Terlevic, D. (2018). Epidemiology of oral mucosal lesions in Slovenia, Radiology and Oncology, 52(3), 263-266. doi: https://doi.org/10.2478/raon-2018-0031

Soluk-Tekkesin M, Wright JM. The World Health Organization Classification ofOdontogenic Lesions: A Summary of the Changes of the 2017 (4th) Edition. TurkPatoloji Derg. 2018;34(1). https://doi.org/10.5146/tjpath.2017.01410 